\title{
TITLE:
}

\section{Black hole radiation with high frequency dispersion}

\section{$\operatorname{AUTHOR}(\mathrm{S})$ :}

Saida, $\mathrm{H}$; Sakagami, M

\section{CITATION:}

Saida, H ... [et al]. Black hole radiation with high frequency dispersion. PHYSICAL REVIEW D 2000, 61(8): 084023.

\section{ISSUE DATE:}

2000-04-15

URL:

http://hdl.handle.net/2433/50233

\section{RIGHT:}

Copyright 2000 American Physical Society 
PHYSICAL REVIEW D, VOLUME 61, 084023

\title{
Black hole radiation with high frequency dispersion
}

\author{
Hiromi Saida* \\ Graduate School of Human and Environmental Studies, Kyoto University, Kyoto 606-8501, Japan \\ Masa-aki Sakagami ${ }^{\dagger}$ \\ Department of Fundamental Sciences, FIHS, Kyoto University, Kyoto 606-8501, Japan \\ (Received 11 May 1999; published 28 March 2000)
}

\begin{abstract}
We consider one model of black hole radiation in which the equation of motion of a matter field is modified to cut off high frequency modes. The spectrum in the model has already been analytically derived in the low frequency range, which has resulted in the Planckian distribution of the Hawking temperature. On the other hand, it has been numerically shown that its spectrum deviates from the thermal one in the high frequency range. In this paper, we analytically derive the form of the deviation in the high frequency range. Our result can qualitatively explain the nature of the numerically calculated spectrum. The origin of the deviation is clarified by a simple discussion.

PACS number(s): 04.70.Dy, 04.62.+v
\end{abstract}

\section{INTRODUCTION}

There are two important theories in modern physics: quantum mechanics and the theory of general relativity. The former has been applied to field theory in flat spacetime and marked triumphs in understanding the wide range of microscopic phenomena. On the other hand, the latter has given us insight into phenomena having close relation to gravity. The theory of general relativity, which is a classical theory, has drastically changed our understanding of space and time. Yet, we have no idea how to treat gravity at the Planck scale. It is unclear whether the unification of quantum mechanics and the theory of general relativity will be accomplished in the context of quantum physics. Today, however, we do not have any idea superior to quantum mechanics in treating Planck scale physics. It is, therefore, meaningful to study how quantum and/or semiclassical effects will be expected in the presence of strong gravity.

The most remarkable discovery including a semiclassical gravitational effect is the Hawking radiation [1], which is concluded by treating matter fields on spacetime as quantum while a black hole metric as classical. According to this theory, a black hole radiates particle flux of a thermal spectrum, whose temperature is $\kappa / 2 \pi$ where $\kappa$ is the surface gravity.

The derivation of the original Hawking radiation relies on the extremely high, over Planck scale, frequency modes. These modes arise from the extremely high gravitational red shift which the radiation undergoes during propagating from the event horizon to the asymptotically flat region. It is the investigation of the Hawking radiation that sheds light on Planck scale physics.

In the study of this physics, one model of a black hole radiation [3-5] has been proposed, which makes an assumption on the effects of unknown Planck scale physics as follows: the equation of motion (EOM) of matter field is modified to have a cutoff of the high frequency modes with

\footnotetext{
*Email address: saida@phys.h.kyoto-u.ac.jp

${ }^{\dagger}$ Email address: sakagami@phys.h.kyoto-u.ac.jp
}

respect to free fall observer. ${ }^{1}$ There are two important properties of this model. The first one is that the modified EOM of matter field violates Lorentz invariance. Secondly, the origin of the black hole radiation we consider in this paper, which is called the mode conversion, ${ }^{2}$ is different from that of the original Hawking radiation. We call the treatment of the black hole radiation in this paper the mode conversion $(M C)$ model hereafter.

The spectrum of the flux in the two dimensional MC model has already been numerically calculated [3]. It has been shown that, although the spectrum almost agrees with the Planckian distributions, it deviates from the thermal one in the high frequency region $\kappa \ll \omega$, where $\omega$ is the energy (frequency) of a massless scalar field. On the other hand, an analytical calculation [4] has resulted in the thermal spectrum, where the analysis has been carried out perturbatively up to lowest orders of $\kappa$ and $\omega$ in the range $\omega<\kappa$. This is appropriate because the derivation of the spectrum in [4] does not take the effects of the high frequency range into account.

The purpose of this paper is to extend the analytical calculation in [4] to the high frequency range, $\kappa<\omega$. We obtain the form of the distribution function, $N(\omega)$, as

$$
N(\omega) \simeq \frac{1-\alpha \omega^{2} / 2}{\exp \left[(2 \pi \omega / \kappa)\left(1-\alpha \omega^{2} / 2\right)\right]-1},
$$

which shows the deviation from the thermal spectrum. Here, $\alpha$ is the square of the cut off scale characterizing the new physics in Planck scale. This result denotes the same tendency as what numerical calculations show. Furthermore, we will give a simple explanation of the occurrence of the deviation.

In Sec. II, we explain the MC model [3]. Section III is

\footnotetext{
${ }^{1}$ This model was motivated by the dumb hole [2], which is a hydrodynamical analogue of a black hole radiation.

${ }^{2}$ The phenomenon called mode conversion has already been known in plasma physics [6-8].
} 
devoted to the calculation of the spectrum. Lastly, we summarize the results in Sec. IV.

Throughout this paper, we use Planck units, $\hbar=c=G$ $=1$, and restrict our discussion in two dimension for simplicity.

\section{MODE CONVERSION (MC) MODEL}

We consider the static spacetime with the metric of the form [3-5]

$$
d s^{2}=-d t^{2}+(d x-v(x) d t)^{2} .
$$

It is obvious that the timelike Killing vector field of this spacetime is $\partial_{t}$. This spacetime has an event horizon at $x_{h}$ satisfying $v\left(x_{h}\right)=-1$. The surface gravity of this spacetime, $\kappa$, is calculated to be $\kappa=(d v / d x)\left(x_{h}\right)$ which is $1 / 4 M$ for the case of the Schwarzschild black hole of mass $M$. Note that the world line of $d x-v(x) d t=0$ is normal to the surface, $t$ $=$ const, and that it is a geodesic of a free fall observer. The velocity of the free fall observer with respect to the rest observer is $v(x)$, where we require $v(x)<0$ and monotone increasing $\partial_{x} v(x)>0$, that is, $v(x \rightarrow \infty) \rightarrow v_{0}$ where -1 $<v_{0} \leqslant 0$ by definition. The proper time of this free fall observer is $t$ of the coordinate (1).

The action of a massless scalar field [3-5] is

$$
S=\frac{1}{2} \int d^{2} x \sqrt{-\mathrm{g}}\left[(\mathbf{u}(\Phi))^{*} \mathbf{u}(\Phi)-(\mathbf{s}(\Phi))^{*} \mathbf{s}(\Phi)\right],
$$

where $\mathrm{g}=\operatorname{det} g_{\mu \nu}, \mathbf{u}$ is a unit tangent to the free fall world line and $\mathbf{s}$ is a unit space-like vector which is outwardpointing and orthogonal to $\mathbf{u}$. We choose the derivatives of $\Phi$ along $\mathbf{u}$ and $\mathbf{s}$ as $\mathbf{u}(\Phi)=u^{\mu} \partial_{\mu} \Phi$ and $\mathbf{s}(\Phi)=\hat{F}\left(s^{\mu} \partial_{\mu}\right) \Phi$, where $\hat{F}\left(s^{\mu} \partial_{\mu}\right)$ is a function of the differential operators, $\partial_{\mu}$, and we require $\hat{F}\left(-s^{\mu} \partial_{\mu}\right)=-\hat{F}\left(s^{\mu} \partial_{\mu}\right)$. This modification, $\hat{F}\left(s^{\mu} \partial_{\mu}\right)$, expresses the MC model's assumption about the effects of unknown Planck scale physics. This leads the EOM of $\Phi$ in the coordinate of Eq. (1) to be

$$
\left(\partial_{t}+v(x) \partial_{x}\right)\left(\partial_{t}+\partial_{x} v(x)\right) \Phi=\hat{F}^{2}\left(\partial_{x}\right) \Phi \text {. }
$$

Hereafter we specify the form of $\hat{F}\left(\partial_{x}\right)$ as $\hat{F}^{2}\left(\partial_{x}\right)=\partial_{x}^{2}$ $+\alpha \partial_{x}^{4}$, where $\alpha=1 / k_{c}^{2}$ and $k_{c}$ is the cut off scale which characterizes the new physics in Planck scale. We set the order of $\alpha$ is of unity, $O(\alpha) \sim 1$.

We require that, at least far from the event horizon, $v(x)$ is nearly constant enough to require the validity of WKB approximation. This means that $\partial_{x} v(x) / k(x) \ll 1$ and $\partial_{x} k(x) / k^{2}(x) \ll 1(k \simeq$ const $)$ far from the event horizon. With setting $\Phi \simeq \exp (-i \omega t+i k x)$, the EOM (3) gives the dispersion relation

$$
\begin{aligned}
& \omega^{\prime}=-v k+\omega, \\
& \omega^{\prime}= \pm \sqrt{k^{2}-\alpha k^{4}} \equiv F(k),
\end{aligned}
$$

where $\omega^{\prime}$ and $\omega$ are the frequencies with respect to the free fall observer and to the rest observer, respectively, since

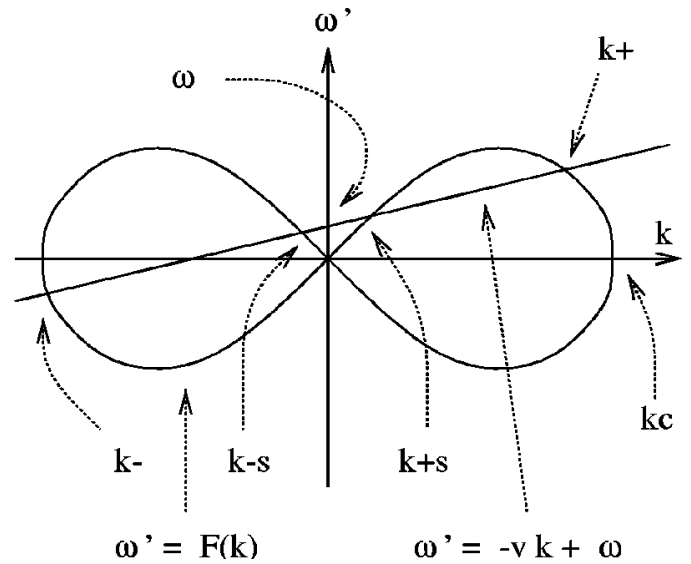

FIG. 1. Dispersion relation of our MC model.

$\mathbf{u}(\Phi)=-i(\omega-v k) \Phi=-i \omega^{\prime} \Phi$ and $\partial_{t} \Phi=-i \omega \Phi$. The Killing frequency, $\omega$, is conserved during time evolution but the free fall frequency, $\omega^{\prime}$, is not. Equations (4) are the dispersion relation of this $\mathrm{MC}$ model, which have four mode solutions for fixed $\omega$. We call the wave numbers of these modes as $k_{-}, k_{-s}, k_{+s}$ and $k_{+}$in increasing order, as shown in Fig. 1. It is not the signature of $\omega$ but of $\omega^{\prime}$ according to which we can judge whether the solution is the positive frequency mode or the negative frequency one [3].

The origin of the black hole radiation in this model can be understood by analyzing a wave packet propagation [3-5]. The group velocity of a wave packet with respect to the free fall observer and the rest observer are expressed as $V_{g}^{\prime}$ $=d \omega^{\prime}(k) / d k$ and $V_{g}=V_{g}^{\prime}+v(x)$ respectively. In the case of $\omega>0$, we can find easily that $k_{-s, \pm}$ modes are of ingoing, while $k_{+s}$ one is of outgoing. In order to understand the physics of mode conversion, the hydrodynamical analogue of black hole radiation is helpful [2,3]. In this model, a fluid flows toward a center, on which we consider a wave packet propagation. Here, $v(x)$ in Eq. (1) represents the velocity of the infalling fluid flow. As shown in Fig. $2, k_{+s}$ mode travels outward away from the event horizon, and against the infalling flow of back ground fluid. In tracing the mode backward in time, it approaches toward the event horizon. In Fig. 3, we

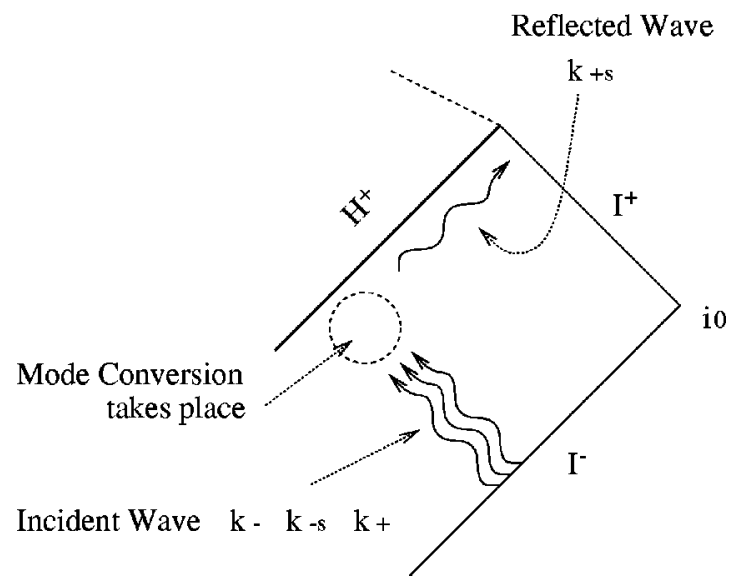

FIG. 2. Schematical description of the mode conversion on an asymptotically flat spacetime. $\mathrm{I}^{ \pm}$are the future and past null infinity. $\mathrm{i}_{0}$ is the spatial infinity. $\mathrm{H}^{+}$is the event horizon. 


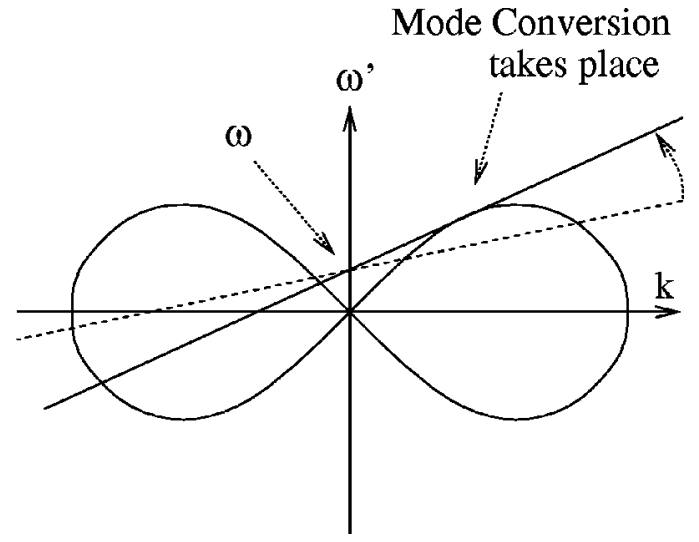

FIG. 3. Graphical description of the mode conversion in the graph of dispersion relation. In view of tracing the wave packet of $k_{+s}$ backward in time, it is converted to the wave packet of $k_{-s, \pm}$.

show how $k_{+s}$ mode moves in the diagram of the dispersion relation. Near the event horizon, its group velocity, $V_{g}^{\prime}\left(k_{+s}\right)$, eventually coincides with the fluid velocity, $v(x)$. Here the mode conversion takes place. In the view of our tracing backward in time, the infalling wave packet of $k_{+s}$ never reaches the event horizon and is converted to the other wave packet of $k_{-s, \pm}$. We should note that the negative free fall frequency mode of $k_{-}$comes to arise in the process of the mode conversion, while the other modes of $k_{-s,+}$ are positive ones.

The situation we consider in Fig. 2, where no transmitted wave acrosses the event horizon, corresponds just to a boundary condition for the calculation of the original Hawking radiation. In the asymptotically flat region, $x \rightarrow \infty$, we can decompose the wave function as

$$
\Phi(x \rightarrow \infty)=\sum_{l= \pm s, \pm} c_{l} \exp \left(-i \omega+k_{l} x\right),
$$

where each coefficient, $c_{ \pm s, \pm}$, can be uniquely determined by this boundary condition. Then we can obtain the form of the black hole radiation spectrum observed at the asymptotically flat region, $N(\omega)$, by Bogoliubov transformation [3]. It becomes

$$
N(\omega)=\left\langle 0_{i}\left|a_{f}^{\dagger} a_{f}\right| 0_{i}\right\rangle=\left|\frac{\omega^{\prime}\left(k_{-}\right) V_{g}\left(k_{-}\right) c_{-}^{2}(\omega)}{\omega^{\prime}\left(k_{+s}\right) V_{g}\left(k_{+s}\right) c_{+s}^{2}(\omega)}\right|,
$$

where $\left|0_{i}\right\rangle$ is the vacuum state of the free fall observer at the initial time $(t \rightarrow-\infty), a_{f}^{\dagger}$ and $a_{f}$ are the creation and annihilation operators of free fall observer at the final time $(t$ $\rightarrow \infty)$. In deriving Eq. (6), we have assumed the Killing frequency spectrum of the wave packet, $\omega(k)$, is sharply peaked around the four wave numbers $k_{ \pm s, \pm}$.

\section{BH RADIATION SPECTRUM IN MC MODEL}

\section{A. Analytical calculation of the spectrum}

\section{The plan to calculate $N(\omega)$}

We consider only the mode function corresponding to the wave number at the peak of $\omega(k)$. We set its form as

$$
\Phi(t, x)=\exp (-i \omega t) \phi(x),
$$

where $\omega$ is the peak value of the Killing frequency spectrum of the wave packet. Then EOM (3) becomes

$$
\alpha \phi^{(4)}+\left(1-v^{2}\right) \phi^{\prime \prime}+2 v\left(i \omega-v^{\prime}\right) \phi^{\prime}+\left(\omega^{2}+i \omega v^{\prime}\right) \phi=0 .
$$

As mentioned at the end of the previous section, we adopt the boundary condition of total reflection, which is expressed as

B.C.: The solution of Eq. (8) dumps with decreasing $x$ inside the event horizon.

We take the origin of spatial coordinate $x$ at the location of the event horizon, that is, $v\left(x_{h}=0\right)=-1$.

To calculate $N(\omega)$, we follow the same plan as in [4]. This plan consists of three steps. The first step is to solve Eq. (8) around the event horizon. We use the Laplace transformation in this step, where the contour of the Laplace integral should be chosen as it satisfies the B.C. Secondly, we look for the solution of Eq. (8) far from the event horizon by means of the WKB approximation. We match the WKB solutions with those of the first step. The existence of the overlap region (matching zone) should be checked, where the solutions in first and second steps are valid. The third step is to calculate $N(\omega)$ at the spatial infinity using Eq. (6).

\section{Formulas for each step and small parameters}

We expand $v(x)$ around the event horizon as

$$
\begin{aligned}
v(x) & \simeq-1+\kappa x, \\
1-v(x)^{2} & \simeq 2 \kappa x,
\end{aligned}
$$

where we require $|\kappa x| \ll 1$ and $\kappa$ is the surface gravity which is assumed to be small itself. We use Laplace transformation of $\phi(x)$,

$$
\phi(x)=\int_{C} d s e^{s x} \widetilde{\phi}(s) .
$$

With approximating every coefficient in EOM (8) up to $O(\kappa)$, we obtain

$$
2 \kappa f(s) \widetilde{\phi}^{\prime}(s)-B(s) \widetilde{\phi}(s)=0,
$$

where $f(s)=s^{2}+i \omega s, \quad B(s)=\alpha s^{4}-\kappa \beta f^{\prime}(s)$ and $\beta=1$ $+i \omega / \kappa$. This gives us

$$
\begin{aligned}
\phi(x)= & \int_{C} d s(s+i \omega)^{i \alpha \omega^{3} / 2 \kappa}\left(s^{2}+i \omega s\right)^{-\beta / 2} \\
& \times \exp \left[s x+\frac{\alpha}{2 \kappa}\left(\frac{1}{3} s^{3}-i \frac{\omega}{2} s^{2}-\omega^{2} s-i \frac{11}{6} \omega^{3}\right)\right] \\
\equiv & \int_{C} d s \exp (h(s))
\end{aligned}
$$

We will find later that the factor $(s+i \omega)^{i \alpha \omega^{3} / 2 \kappa}$, which is ignored in [4], will make important contributions to the deviation of the $\mathrm{MC}$ spectrum from the thermal one. 


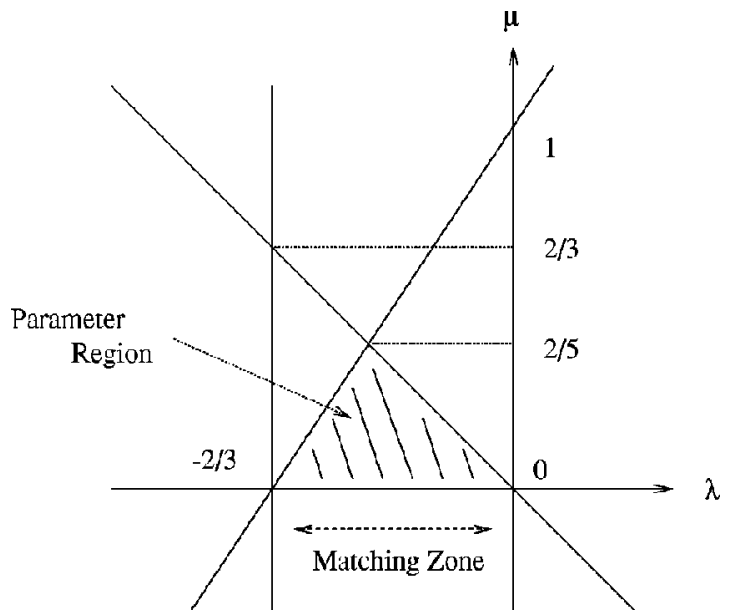

FIG. 4. Parameter region we consider.

For WKB approximation, we set

$$
\phi(x)=\exp \left[i \int_{x_{0}}^{x} k\left(x^{\prime}\right) d x^{\prime}\right],
$$

where $x_{0}$ is a constant. We expand $k(x)=k_{0}(x)$ $+(1 / \lambda) k_{1}(x)+\cdots$ in introducing the scaling $x \rightarrow \lambda x\left(\partial_{x}\right.$ $\left.\rightarrow(1 / \lambda) \partial_{x}\right)[4]$, then we obtain from Eq. (8) that

$$
\begin{gathered}
O(1): \alpha k_{0}^{4}-\left(1-v^{2}\right) k_{0}^{2}-2 \omega v k_{0}+\omega^{2}=0 \\
O\left(\lambda^{-1}\right): k_{1}=\frac{i}{2} \frac{d}{d x} \ln \left[\sqrt{\alpha}\left(2 \alpha k_{0}^{3}-\left(1-v^{2}\right) k_{0}-\omega v\right)\right],
\end{gathered}
$$

where four solutions of $k$ correspond to $k_{ \pm, \pm s}$.

To calculate $N(\omega)$, we should evaluate $\omega^{\prime} V_{g}$. This is given by Eq. (4) to be

$$
\omega^{\prime} V_{g}=-2 \alpha k_{0}^{3}+\left(1-v_{0}^{2}\right) k_{0}+\omega v_{0} .
$$

As stated in Sec. I, $N(\omega)$ deviates from the thermal spectrum in the high frequency region $\kappa<\omega$ [3]. So we may not be able to derive the deviation with expanding $k_{0}$ around $\omega$ $=0$ as done in [4]. Thus we focus our attention on the region, $\kappa<\omega$, in order to derive the deviation. It is expected that the deviation becomes visible at the value of $\omega$ which does not exceed $\kappa$ so much. Thus, when we analyze how the MC spectrum deviates from the thermal one, the fourth term in the left-hand side of Eq. (14) can be neglected in obtaining WKB solutions. This means we require

$$
\alpha k_{0}^{4} \gg \omega^{2}, \quad\left(1-v^{2}\right) k_{0}^{2} \gg \omega^{2}, \quad \omega v k_{0} \gg \omega^{2} .
$$

The other restrictions on the region of $\kappa$ and $\omega$ come from the validity of the solution of WKB and that of the Laplace transformation. These restrictions are expressed by $\left|k_{1} / k_{0}\right|$ $\ll 1$ from $\mathrm{WKB}$ and by $\kappa x \ll 1$ and $\left|s_{0} x\right| \gg 1$ from Laplace transformation, where $s_{0}$ is a saddle point of integrand in Eq. (12) in using the asymptotic expansion of $x$ by the method of steepest descent contour (SDC). We can obtain the matching zone from these inequalities. It becomes

$$
\left(\frac{\alpha}{\kappa}\right)^{1 / 3} \ll x \ll \frac{1}{\kappa},
$$

where we use the forms of $s_{0}$ and $k_{0,1}$ which are calculated later in Appendix A and at Eq. (23). Furthermore, we require $\omega x \ll 1$ for later convenience.

With introducing $\lambda$ and $\mu$ by $\kappa x=(1 / \kappa \sqrt{\alpha})^{\lambda}$ and $\omega$ $=\kappa(1 / \kappa \sqrt{\alpha})^{\mu}$ where $\kappa \sqrt{\alpha} \ll 1$, the parameter region we discussed above can be expressed as

$$
3 \lambda>2(\mu-1), \quad-\frac{2}{3}<\lambda<0, \quad 0<\mu, \quad \lambda+\mu<0,
$$

where first and second inequalities obtained by Eq. (17) with Eqs. (9) and (18) respectively.

\section{Calculation of $N(\omega)$}

We can proceed to calculate $N(\omega)$ with the above preparations. As we carry out the Laplace integral of Eq. (12) in Appendix A, we obtain the solution of Eq. (8) in the matching zone as

$$
\phi(x)=\phi_{1}(x)+\phi_{2}(x)+\phi_{3}(x),
$$

where, as demonstrated below, $\phi_{1,2,3}$ should be matched with WKB solutions, $\phi_{ \pm,+s}$, respectively. These are expressed as

$$
\begin{aligned}
\phi_{1,2}(x)= & \sqrt{\pi} e^{i \pi(n+1 / 2-\varepsilon 3 / 4)-i 11 \alpha \omega^{3} / 12 \kappa} e^{\varepsilon \pi \omega / 2 \kappa\left(1-\alpha \omega^{2} / 2\right)}\left(\frac{2 \kappa}{\alpha}\right)^{-1 / 4-i \omega / 2 \kappa+i \alpha \omega^{3} / 4 \kappa} x^{-3 / 4-i \omega / 2 \kappa} \\
& \times \exp \left[\varepsilon i \frac{2}{3} \sqrt{\frac{2 \kappa}{\alpha}} x^{3 / 2}+i \frac{\omega}{2} x\right] \\
\phi_{3}(x)= & e^{-5 \xi / 3} 2 \sinh \left[\frac{\pi \omega}{\kappa}\left(1-\frac{\alpha \omega^{2}}{2}\right)\right] \Gamma\left[-i \frac{\omega}{\kappa}\left(1-\frac{\alpha \omega^{2}}{2}\right)\right] x^{i \omega / \kappa},
\end{aligned}
$$

where $(\varepsilon, n)=(-1,0),(1,1)$ for $\phi_{1}$ and $\phi_{2}$ respectively and $\xi=i \alpha \omega^{3} / 4 \kappa$. The factor $1-\alpha \omega^{2} / 2$ shows the difference form the analysis in [4], which arises from the factor $(s+i \omega)^{i \alpha \omega^{3} / 2 \kappa}$ in the integrand of Eq. (12). 
Next we shift our step to obtaining WKB solutions. We can obtain $k_{0}$ from Eq. (14) with the conditions of Eq. (17) only. Then $k_{0}$ becomes

$$
k_{0} \simeq \begin{cases}\omega /(1+v) & \text { for } k_{+s}, \\ \pm \sqrt{\left(1-v^{2}\right) / \alpha}+v \omega /\left(1-v^{2}\right) & \text { for } k_{ \pm},\end{cases}
$$

where these forms of $k_{0}$ are valid in the region $3 \lambda>2(\mu-1)$ which is given by Eq. (17). Thus, for the purpose of matching with Eq. (20), we obtain WKB solutions in the parameter region (see Fig. 4) of Eq. (19) as

$$
\begin{aligned}
& \phi_{ \pm}(x) \simeq A_{ \pm}( \pm 2 \kappa)^{-3 / 4} x^{-3 / 4-i \omega / 2 \kappa} \exp \left[ \pm i \frac{2}{3} \sqrt{\frac{2 \kappa}{\alpha}} x^{3 / 2}+i \frac{\omega}{2} x\right] \\
& \phi_{+s}(x) \simeq A_{+s} x^{i \omega / \kappa}
\end{aligned}
$$

where $A_{ \pm,+s}$ are the coefficients obtained by $x_{0}$ in Eq. (13).

By matching WKB solutions, Eqs. (24) and (25), with that of Laplace transformation, Eq. (20), we obtain the wave function as

$$
\begin{aligned}
\phi(x)= & \sqrt{\pi} e^{i 9 \pi / 4-11 \xi / 3} e^{-\pi \omega / 2 \kappa\left(1-\alpha \omega^{2} / 2\right)}\left(\frac{2 \kappa}{\alpha}\right)^{-1 / 4-i \omega / 2 \kappa+\xi}\left[\frac{(-2 \kappa)^{3 / 4}}{A_{-}} \phi_{-}(x)+e^{-i 5 \pi / 2+\pi \omega / \kappa\left(1-\alpha \omega^{2} / 2\right)} \frac{(2 \kappa)^{3 / 4}}{A_{+}} \phi_{+}(x)\right] \\
& +e^{-5 \xi / 3} 2 \sinh \left[\frac{\pi \omega}{\kappa}\left(1-\frac{\alpha \omega^{2}}{2}\right)\right] \Gamma\left[-i \frac{\omega}{\kappa}\left(1-\frac{\alpha \omega^{2}}{2}\right)\right] \frac{1}{A_{+s}} \phi_{+s}(x) .
\end{aligned}
$$

Note that we can use this result beyond the matching zone, i.e. $(\alpha / \kappa)^{1 / 3} \ll x$ which is given by the validity of WKB approximaton, $\left|k_{1} / k_{0}\right| \ll 1$.

To obtain $N(\omega)$, we need to evaluate $\omega^{\prime} V_{g}$ and $\phi(x)$ at the asymptotically flat region. We obtain with taking the limit $v(x \rightarrow \infty) \rightarrow v_{0}$ that

$$
\begin{aligned}
& \phi_{ \pm}(x \rightarrow \infty) \simeq A_{ \pm}( \pm 1)^{-1 / 2}\left(1-v_{0}^{2}\right)^{-3 / 4} \exp \left[ \pm i \sqrt{\frac{1-v_{0}^{2}}{\alpha} x}\right. \\
&\left.+i \frac{v_{0} \omega}{1-v_{0}^{2}} x\right] \\
& \phi_{+s}(x \rightarrow \infty) \simeq A_{+s} \exp \left[-i \frac{2 v_{0} \omega}{1-v_{0}^{2}} x\right]
\end{aligned}
$$

and

$$
\begin{aligned}
\omega^{\prime}\left(k_{ \pm}\right) V_{g}\left(k_{ \pm}\right) & \simeq \mp \frac{\left(1-v_{0}^{2}\right)^{3 / 2}}{\sqrt{\alpha}}, \\
\omega^{\prime}\left(k_{+s}\right) V_{g}\left(k_{+s}\right) & \simeq-\omega .
\end{aligned}
$$

Then we get $c_{-}$and $c_{+s}$ by Eqs. (26), (27) and (28), and finally obtain $N(\omega)$ by Eq. (6) to be

$$
N(\omega)=\frac{1-\alpha \omega^{2} / 2}{\exp \left[(2 \pi \omega / \kappa)\left(1-\alpha \omega^{2} / 2\right)\right]-1} .
$$

This result shows that MC spectrum is enhanced in comparison with Hawking spectrum.

\section{B. Comparison with numerical results}

We compare the analytically derived form of the spectrum, Eq. (31), with numerical calculations. The numerical calculations are carried out with two types of $v(x)$, which are shown successively in the following two subsections.

We have used MATHEMATICIA to solve Eq. (8) and fit its solution with the form, $\phi=\Sigma_{l= \pm, \pm S} c_{l} \exp \left(i k_{l}\right)$, in order to get $c_{-}$and $c_{+s}$.

Note that we have set $\kappa / 2 \pi=0.0008$ and $\alpha=1$ throughout these numerical computations.

\section{First type of $v(x)$ : CJ type}

First example of $v(x)$, which we call Corley-Jacobson (CJ) type [3], takes the form

$$
v(x)=\operatorname{sgn}(x) \frac{1}{2} \sqrt{\tanh \left[(2 \kappa x)^{2}\right]}-1
$$

To let $k_{-}$mode be a negative free fall frequency one at the asymptotically flat region, the value of $\omega$ is restricted by upper value $\omega_{\max } \sim 0.16$. Further, in our parameter region (19), the range of $\omega$ is bounded as $0.005<\omega<0.04 \quad(0<\mu$ $<2 / 5)$.

We have calculated the relative deviation,

$$
R(\omega)=\frac{N(\omega)}{N_{H}(\omega)}-1
$$

where $N_{H}$ is the Hawking spectrum. Figure 5 is the plot of this relative deviation. $R(\omega)$ grows qualitatively more and more with increasing $\omega$. 


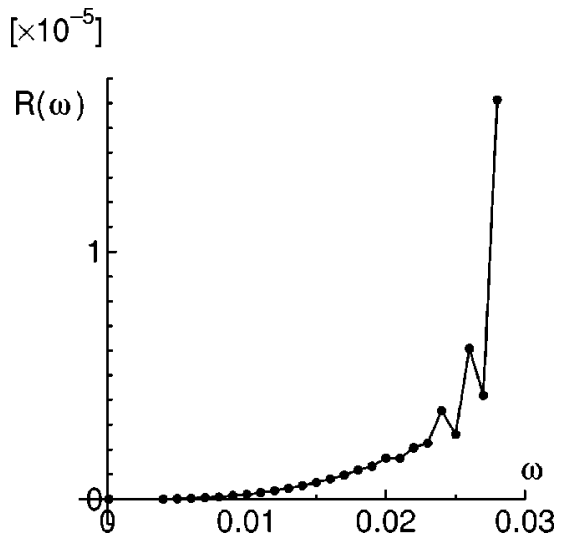

FIG. 5. Graph of relative deviation, $R(\omega)$, with CJ type of $v(x)$. $R(\omega)$ grows qualitatively more and more with increasing $\omega$.

This shows that the MC spectrum is enhanced in comparison with the Hawking spectrum, as is indicated by the analytical result (31). However Fig. 8, which is the plots of the logarithm of relative deviations, exhibit that the order of $R(\omega)$ in CJ type differs largely from our result (31). We expect that this difference can be reduced by changing the form of $v(x)$. The clue of this change is in the EOM (8). The most effective term for determining the analyticity of the solution of Eq. (8) is the second term, $\left(1-v^{2}\right) \phi^{\prime \prime} .^{3}$ Thus, in order to compare Eq. (31) with a numerical calculation, the expansion $1-v^{2} \simeq 2 \kappa x$ near the horizon should be better than the expansion $v \simeq-1+\kappa x$. By the way, $v(x)$ of $\mathrm{CJ}$ type can be expanded near horizon as

$$
\begin{aligned}
v(x) & \simeq-1+\kappa x+O\left((\kappa x)^{4}\right), \\
1-v(x)^{2} & \simeq 2 \kappa x+O\left((\kappa x)^{2}\right) .
\end{aligned}
$$

While CJ type is a good form for the expansion $v \simeq-1$ $+\kappa x$ near the horizon, it is not good for the expansion 1 $-v^{2} \simeq 2 \kappa x$. We should modify $v(x)$ to let the expansion 1 $-v^{2} \simeq 2 \kappa x$ near the horizon be better than CJ type.

\section{Second type of $v(x): D S$ type}

The modified type of $v(x)$, which is called DoubleSquareroot-Tanh (DS) type hereafter, is

$$
v(x)=-\sqrt{1-\operatorname{sgn}(x) \frac{3}{4} \sqrt{\tanh \left[\left(\frac{8}{3} \kappa x\right)^{2}\right]}} .
$$

The range of $\omega$ is $0.005<\omega<0.04(0<\mu<2 / 5)$, because of the same reason as that of CJ type. Figure 6 shows the shapes of $v(x)$ of CJ and DS types. They vary from -1 at $x=0$ to $x=-1 / 2$ at $x \rightarrow \infty$.

The $v(x)$ of DS type takes the form near horizon:

$$
v(x) \simeq-1+\kappa x+O\left((\kappa x)^{2}\right),
$$

\footnotetext{
${ }^{3}$ This is also implied in Sec. IV.
}

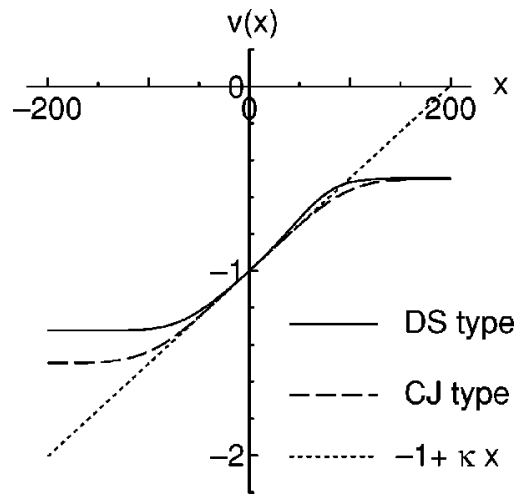

FIG. 6. Shapes of $v(x)$ in the form of CJ and DS types. They vary from -1 at $x=0$ to $x=-1 / 2$ at $x \rightarrow \infty$.

$$
1-v(x)^{2} \simeq 2 \kappa x+O\left((\kappa x)^{5}\right) .
$$

The expansion $v \simeq-1+\kappa x$ near horizon for CJ type is better than that for DS type, however the expansion $1-v^{2} \simeq 2 \kappa x$ for DS type is better than that for CJ type.

Figure 7 is the plot of the relative deviation for DS type. $R(\omega)$ grows qualitatively more and more with increasing $\omega$. This figure and Fig. 5 implies that the details of the MC spectrum depend strongly on the form of $v(x)$. The total behavior of Fig. 7, however, shows that, althogh some data take negative value, the qualitative nature of the MC spectrum is enhancement in comparison with the Hawking spectrum, which is indicated by the analytical result (31). Then Fig. 8 involves the plot of the absolute value of $R(\omega)$ in DS type. This shows that the order of $R(\omega)$ can be explained by our analytical result (31) fairly well, since the higher order effects by $1-v^{2}$ in DS type are weakened to agree with the assumption made in our analytical treatment of the MC spectrum.

From above it is valid to mention that our resultant spectrum (31) can explain the qualitative nature of the MC spectrum in the range, $\kappa<\omega<\kappa(\kappa \sqrt{\alpha})^{-2 / 5}$.

\section{SUMMARY AND DISCUSSION}

We have derived the correction to the black hole radiation spectrum in the MC model in the frequency range, $0<\mu$ $<2 / 5, \omega=\kappa(1 / \kappa \sqrt{\alpha})^{\mu}$. The MC model cut the high frequency mode off, so one may guess naively that the spec-

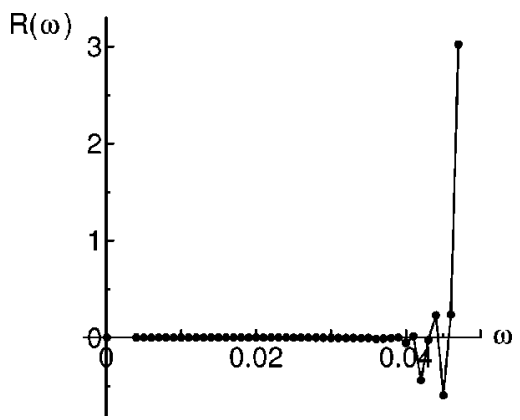

FIG. 7. Graph of relative deviation, $R(\omega)$, with DS type of $v(x) . R(\omega)$ grows qualitatively more and more with increasing $\omega$. 


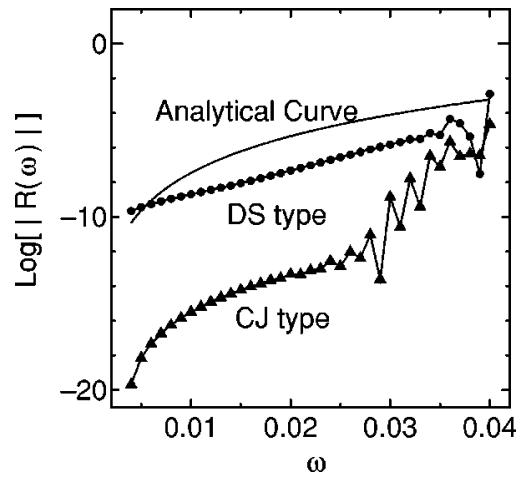

FIG. 8. Graphs of logarithm of absolute value of the relative deviations. The solid line is for our analytical result. These show that our resultant spectrum explains the qualitative nature of the MC spectrum.

trum would be suppressed. Our result, however, shows that the $\mathrm{MC}$ spectrum is enhanced in the frequency range, $0<\mu$ $<2 / 5$. This is consistent with the numerical calculations as seen above.

The deviation factor $1-\alpha \omega^{2} / 2$ in Eq. (31) is understood as a correction of red shift which outgoing modes undergo during propagating from the event horizon to the asymptotically flat region. This is reflected on the form of $k_{+s}$ mode, $x^{i(\omega / \kappa)\left(1-\alpha \omega^{2} / 2\right)}$ in Eq. (A6). This form can be easily explained. Neglecting the fourth term in Eq. (8) which is of second order of small quantities $\kappa$ and $\omega$, we obtain

$$
\alpha \varphi^{\prime \prime \prime}+2 \kappa x \varphi^{\prime}-2(1-\kappa x)(i \omega-\kappa) \varphi=0,
$$

where we set $\phi^{\prime}(x)=\varphi(x)$ and $v(x) \simeq-1+\kappa x$. Under the assumption $\alpha<1$, we expand $\varphi=\varphi_{0}+\alpha \varphi_{1}+\cdots$ which leads to

$$
\begin{aligned}
& 2 \kappa x \varphi_{0}^{\prime}-2(1-\kappa x)(i \omega-\kappa) \varphi_{0}=0, \\
& 2 \kappa x \varphi_{1}^{\prime}-2(1-\kappa x)(i \omega-\kappa) \varphi_{1}=-\varphi_{0}^{\prime \prime \prime} .
\end{aligned}
$$

The lowest order solution is given by

$$
\varphi_{0}(x)=x^{i \omega / \kappa-1} e^{(\kappa-i \omega) x} .
$$

We are interested in the analyticity of the following form:

$$
\varphi_{0} \sim x^{i \omega / \kappa-1} \Rightarrow \phi_{0} \sim x^{i \omega / \kappa}
$$

since the temperature of the original Hawking radiation is determined by the form of an outgoing mode which takes the same form as above. Then we consider the correction to this analyticity. With setting $B=i \omega / \kappa-1$, we obtain $\varphi_{1}$ as

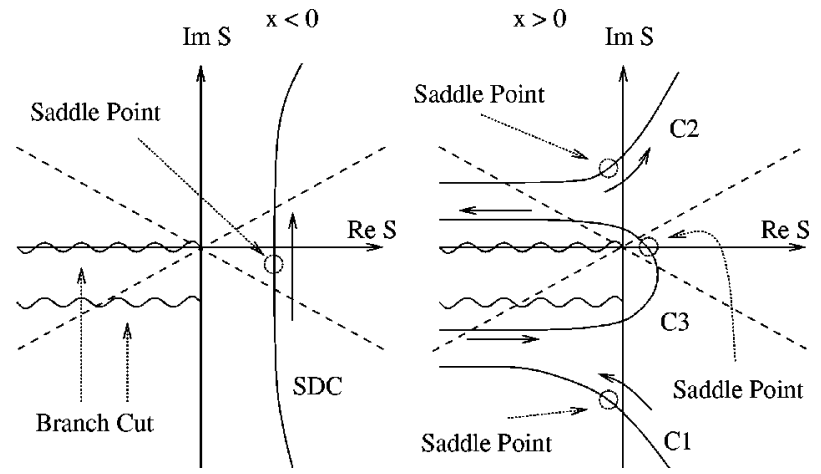

FIG. 9. Contour of Laplace integral satisfying B.C. Left figure is for $x<0$. Right figure is for $x>0$.

$$
\begin{aligned}
\varphi_{1}(x)= & -\frac{\alpha}{2}\left[-\frac{B^{3}-3 B^{2}+2 B}{3 \kappa x^{3}}+\frac{3\left(B^{3}-B^{2}\right)}{2 x^{2}}-\frac{3 \kappa B^{3}}{x}\right. \\
& \left.-\kappa^{2} B^{3} \ln x\right] \varphi_{0}(x) .
\end{aligned}
$$

We can recognize the fourth term in the square brackets as the secular one. Although this term is not dominant, it can change the analyticity which determines the temperature of the radiation. We use the renormalization group method in order to take all of the contributions of the secular term of $\varphi_{1}$ into account $[9,10]$. In the parameter region $\omega / \kappa \geqslant 1$, we obtain

$$
\begin{aligned}
& \varphi \sim x^{\alpha(i \omega-\kappa)^{3} / 2 \kappa+i \omega / \kappa-1} \sim x^{\alpha(i \omega)^{3} / 2 \kappa+i \omega / \kappa-1} \\
\Rightarrow & \phi \sim x^{i(\omega / \kappa)\left(1-\alpha \omega^{2} / 2\right)}
\end{aligned}
$$

which shows the same deviation as in Eq. (31).

Further, our result agrees with that of [11]. In the paper [11], the order of the MC spectrum's deviation has been estimated, which is consistent with our spectrum, $N(\omega)$.

\section{ACKNOWLEDGMENTS}

We would like to thank J. Soda for useful discussions. This work was supported in part by Monbusho Grant-in-Aid for Scientific Research No. 10640258. One of us (H.S.) also thanks S. Kawai for his helpful comments.

\section{APPENDIX: CALCULATION OF LAPLACE INTEGRAL}

We approximate Eq. (12) in this appendix by an asymptotic expansion of $x$ with the method of steepest descent contour (SDC). The saddle points, $s_{0}$, are the solutions of $d h / d s=0$ :

$$
\alpha s_{0}^{4}+2 \kappa x s_{0}^{2}+2(i \omega \kappa x-\kappa \beta) s_{0}-i \omega \kappa \beta=0 .
$$

The $\phi(x)$ approximated by SDC is expressed as

$$
\phi(x) \simeq \sqrt{2 \pi}\left(|x| h^{\prime \prime}\left(s_{0}\right)\right)^{-1 / 2} \exp \left[x h\left(s_{0}\right)+i \pi\left(\frac{1}{2} \gamma+n\right)\right],
$$



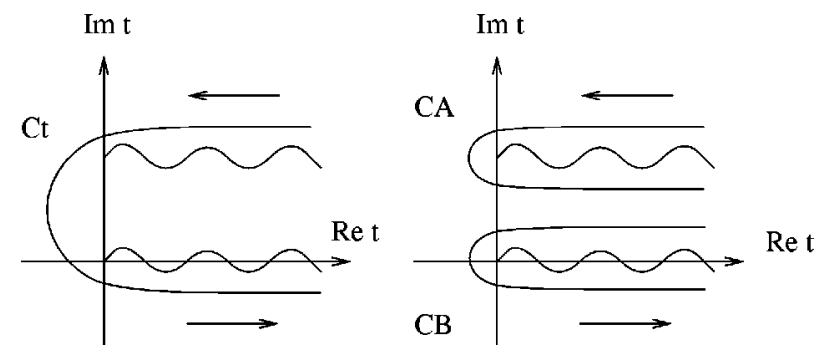

FIG. 10. Contour of Laplace integral for $\phi_{3}(x)$.

where $n$ is the integer determining the direction of the SDC and $\gamma=0,1$ for $x<0$ and $x>0$ respectively.

With approximating Eqs. (A1) and (A2) in the paramater region (19), we obtain the saddle point satisfying B.C. in $x$ $<0$ as

$$
s_{0} \simeq \sqrt{2 \kappa|x| / \alpha}-i \beta / 2|x|,
$$

and, for $x>0$, the saddle points are

$$
s_{0} \simeq\left\{\begin{array}{l}
\beta / x, \\
\pm i \sqrt{2 \kappa x / \alpha}-\beta / 2 x .
\end{array}\right.
$$

The integral contour is determined as shown in Fig. 9, where the contour should asymptote to one of the three regions, $\pi / 6<\arg (s)<\pi / 2,5 \pi / 6<\arg (s)<7 \pi / 6$ and $3 \pi / 2<\arg (s)$ $<11 \pi / 6$ since, in the limit $|s| \rightarrow \infty$, the convergence of Eq. (12) requires $\operatorname{Re}\left(s^{3}\right)<0$. The waving curves are the branch cuts of the integrand in Eq. (12).

The SDC of saddle point $\beta / x$ approximates the contribution from the contour $C_{3}$. We can find, however, that this contribution is obtained by integral representations of some special functions more correctly than by the SDC approximation. So we use SDC approximation only for the contributions from the contours $C_{1}$ and $C_{2}$. The dominant dependence on $x$ of these contributions is obtained by Eq. (A2) to give us Eq. (21).
To carry out the contour integral of $C_{3}$, we transform $s$ to $t$ by $s x=e^{-i \pi} t$. While we choose the argument of $s$ as $-\pi$ $<\arg (s)<\pi$, the argument of $t$ is to be $0<\arg (t)<2 \pi$. Then Eq. (12) becomes

$$
\begin{aligned}
\phi_{3}(x)= & e^{-i \pi-i 11 \alpha \omega^{3} / 12 \kappa}(i \omega)^{-\beta / 2+i \alpha \omega^{3} / 2 \kappa} x^{\beta / 2-1} \\
& \times \int_{C_{t}} d t(-t)^{-\beta / 2}\left(1+\frac{t}{-i \omega x}\right)^{-\beta / 2+i \alpha \omega^{3} / 2 \kappa} \\
& \times \exp \left[-t+\frac{\alpha}{2 \kappa}\left(-\frac{t^{3}}{3 x^{3}}-i \frac{\omega t^{2}}{2 x^{2}}+\frac{\omega^{2} t}{x}\right)\right],
\end{aligned}
$$

where $C_{t}$ is the contour on $t$-plane corresponding to $C_{3}$, and $i \omega=e^{i \pi / 2} \omega$ and $-i \omega x=e^{i 3 \pi / 2} \omega x$. We can simplify the exponent of Eq. (A5) to be $\exp (-t)$ in our parameter region (19) by the same argument in [4]: by $1 /\left|\kappa x^{3}\right| \ll 1$ and $\omega x$ $\ll 1$, the correction by $\exp [(\alpha / 2 \kappa)(\cdots)] \simeq 1+(\alpha / 2 \kappa)(\cdots)$ is negligible. With dividing the contour $C_{t}$ into $C_{A}$ and $C_{B}$ as shown in Fig. 10 and using the integral representation of Whittaker function, we can express Eq. (A5) as the sum of two Whittaker functions.

Further we can simplify it by one confluent hypergeometric function to be

$$
\begin{aligned}
\phi_{3}(x)= & e^{-11 \xi / 3} 2 \sinh \left[\frac{\pi \omega}{\kappa}\left(1-\frac{\alpha \omega^{2}}{2}\right)\right] \Gamma\left[-i \frac{\omega}{\kappa}\left(1-\frac{\alpha \omega^{2}}{2}\right)\right] \\
& \times e^{-i \omega x} x^{i \omega / \kappa\left(1-\alpha \omega^{2} / 2\right)} \\
& \times F\left[\frac{\beta}{2}, 1+i \frac{\omega}{\kappa}\left(1-\frac{\alpha \omega^{2}}{2}\right) ; i \omega x\right],
\end{aligned}
$$

where $\xi=i \alpha \omega^{3} / 4 \kappa, \quad \beta=1+i \omega / \kappa, \Gamma$ is the gamma function and $F$ is the confluent hypergeometric function. Then we obtain the dominant dependence on $x$ of $\phi_{3}$ (see Fig. 10), which becomes Eq. (22).
[1] S. W. Hawking, Commun. Math. Phys. 43, 199 (1975).

[2] W. G. Unruh, Phys. Rev. D 51, 2827 (1995).

[3] S. Corley and T. Jacobson, Phys. Rev. D 54, 1568 (1996).

[4] S. Corley, Phys. Rev. D 57, 6280 (1998).

[5] S. Corley and T. Jacobson, Phys. Rev. D 57, 6269 (1998).

[6] D. G. Swanson, Plasma Waves (Academic, New York, 1989).

[7] T. H. Stix, Waves in Plasmas (American Institute of Physics, New York, 1992).
[8] D. G. Swanson, Theory of Mode Conversion and Tunneling in Inhomogeneous Plasmas (John Wiley \& Sons, New York, 1998).

[9] L. Y. Chen, N. Goldenfeld, and Y. Oono, Phys. Rev. Lett. 73, 1311 (1994).

[10] L. Y. Chen, N. Goldenfeld, and Y. Oono, Phys. Rev. E 54, 376 (1996).

[11] Y. Himemoto and T. Tanaka, Phys. Rev. D 61, 064004 (2000). 